\title{
Back out of Locking Pin with Hinge Fracture after High Tibial Osteotomy
}

\author{
Shuhei Otsuki, MD, Tomohiko Murakami, MD, Rei Morikura, MD, Kosuke Nakagawa, MD, \\ Yoshinori Okamoto, MD, and Masashi Neo, MD \\ Department of Orthopedic Surgery, Osaka Medical College, Osaka, Japan
}

Low-profile fixation devices for medial opening wedge high tibial osteotomy (OWHTO) were developed in order to avoid skin irritation and additional invasion. However, the low-profile system is associated with additional risks. We report three cases of locking pin back out with the lowprofile locking plate system for medial OWHTO.

Keywords: Knee, Osteotomy, Hinge fracture, Locking plate

Medial opening wedge high tibial osteotomy (OWHTO) is a well-established treatment for varus malalignment with medial compartment osteoarthritis ${ }^{1)}$, and advancements in the lockingplate system have further improved the clinical outcome especially in the case of TomoFix (DePuy Synthes, Solothurn, Switzerland $)^{2-4)}$. FlexitSystem (Neosteo, Nantes, France), which has been used especially in Europe, has the advantage of an anatomical low-profile design with the locking screw-pin system. We performed 15 consecutive OWHTO using this plate from July 2014 to March 2015; however, back out of the pin at the proximal tibia was seen in three patients. The objectives of this report were to introduce the locking pin back out after OWHTO and elucidate the failure mechanism.

Received June 4, 2017; Revised July 6, 2017; Accepted July 11, 2017

Correspondence to: Shuhei Otsuki, MD

Department of Orthopedic Surgery, Osaka Medical College, 2-7

Daigakumachi Takatsuki, Osaka 569-8686, Japan

Tel: +81-72-683-1221, Fax: +81-72-683-8553

E-mail: ort182@osaka-med.ac.jp

This is an Open Access article distributed under the terms of the Creative Commons Attribution Non-Commercial License (http://creativecommons.org/licenses/by-nc/4.0/) which permits unrestricted non-commercial use, distribution, and reproduction in any medium, provided the original work is properly cited.

\section{Case Reports}

\section{Case 1}

A 74-year-old female with a history of medial knee joint pain for 5 years underwent OWHTO using FlexitSystem. OWHTO was performed with a 9-mm opening biplane osteotomy ${ }^{2}$ filling with $\beta$-tricalcium phosphate (Olympus, Tokyo, Japan) and placing the FlexitSystem plate anteromedial to the tibia for fixation per the manufacturer's protocol. According to the radiographic analysis, the preoperative hip-knee-ankle angle (HKA) improved from $5^{\circ}$ of varus to $4^{\circ}$ of valgus. The percentage of mechanical axis (\%MA) and medial proximal tibial angle (MPTA) were altered between the preoperative and postoperative examinations from $30 \%$ to $63 \%$ and from $87^{\circ}$ to $95^{\circ}$, respectively. Posterior tibial slope (PTS) was $5^{\circ}$, which showed no change after OWHTO (Fig. 1A-D). Rehabilitation was started with $50 \%$ of weight bearing from postoperative day 1 . Following the initiation of rehabilitation program, a type III hinge fracture with back out of the locking pin at the proximal tibia was detected 2 weeks after surgery (Fig. 1C-F). A second surgery was performed to replace the locking pins with screws at the proximal tibia and to fix the type III fracture with cannulated screws from the lateral tibia (Fig. 1G-I). No correction loss was detected at the final follow-up. 

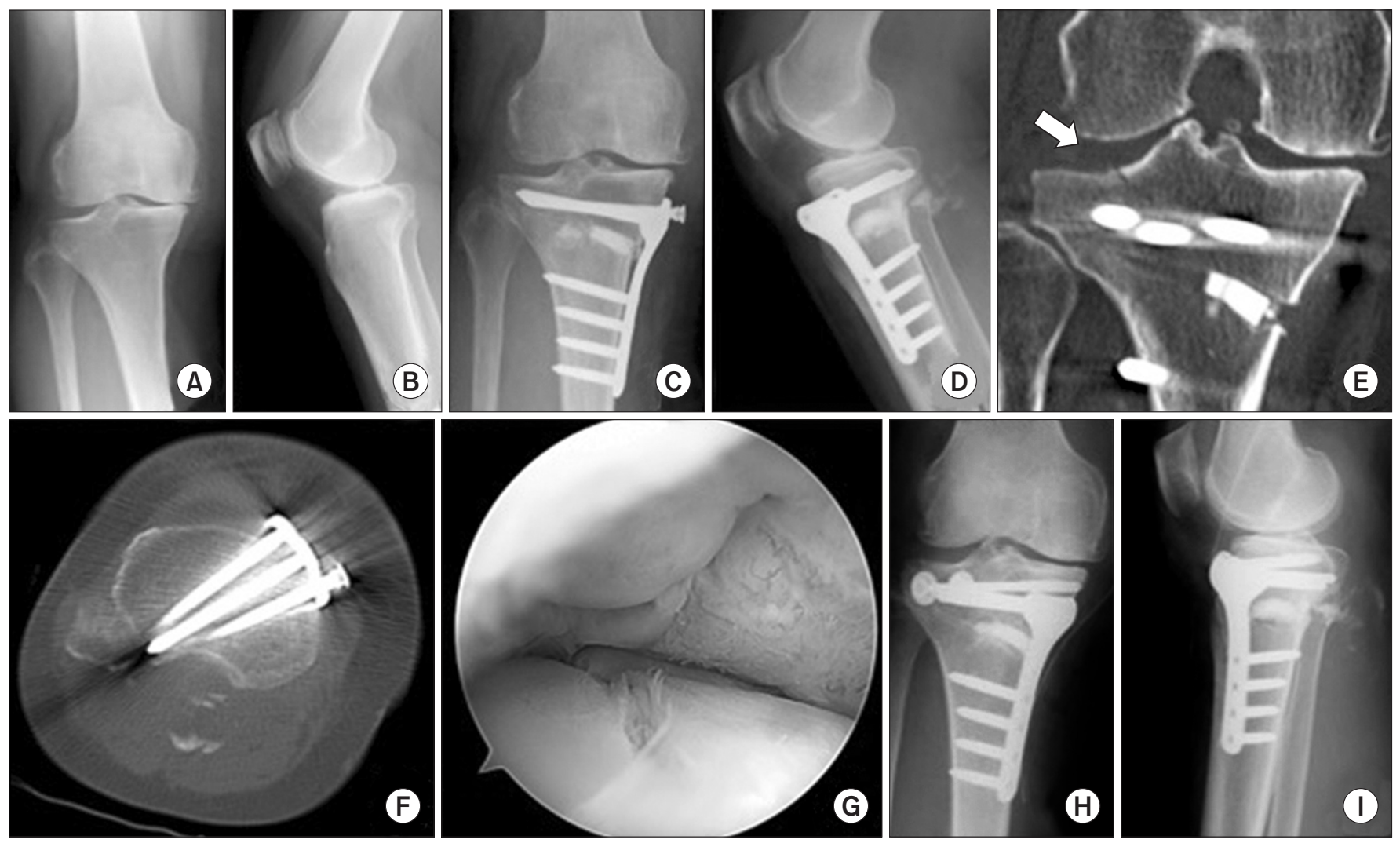

Fig. 1. (A, B) Preoperative X-rays of a 74-year-old female (case 1). (C, D) X-rays at postoperative week 2. (E, F) Type III hinge fracture in computed tomography. $(\mathrm{G})$ Arthroscopic detection of type III hinge fracture. $(\mathrm{H}, \mathrm{I})$ Hinge fracture fixation performed in second surgery.

\section{Case 2}

A 65-year-old female with a prolonged history of medial knee pain underwent OWHTO with FlexitSystem. OWHTO was performed with a 9-mm opening biplane osteotomy filling with $\beta$ tricalcium phosphate. HKA improved from $4^{\circ}$ of varus to $5^{\circ}$ of valgus. The $\% \mathrm{MA}$ and MPTA were altered from $30 \%$ to $65 \%$ and from $83^{\circ}$ to $91^{\circ}$, respectively. The PTS was $8^{\circ}$, which showed no change after OWHTO (Fig. 2A-D). Rehabilitation was initiated from postoperative day 1 with $50 \%$ of weight bearing and then full weight bearing from day 14. A type III hinge fracture with back out of the proximal locking pin was detected at 3 weeks after surgery (Fig. 2E-G). Type III fracture was treated with low intensity pulsed ultrasound to accelerate bone healing without weight bearing. Locking pin deviation progressed at 6 weeks after surgery. Finally, the backed out pin was removed without any correction loss.

\section{Case 3}

A 68-year-old male underwent OWHTO with a type I fracture (Fig. 3A and B). One month after surgery, the locking pin did not seem to have backed out, and he could walk with full-weight bearing without pain (Fig. 3C). Two months after surgery, the center pin at the proximal plate backed out (Fig. 3D), and the proximal posterior screw had also backed out at 15 months after surgery (Fig. 3E and F).

\section{Discussion}

Although there has been a continual evolution in the hardware, OWHTO has a relatively high rate of fixation failure ${ }^{5-77}$. Martin et al. ${ }^{8)}$ reported the rates of adverse events after OWHTO in a sample of more than 300 cases. Of these, $83 \%$ were with a non-locking plate and $17 \%$ with a locking plate. Hardware failure occurred in $4 \%$ of cases, including $2.7 \%$ with a broken screw and $1.3 \%$ with back out of the pin, with no loss of correction and additional surgery. We experienced three cases of locking pin back out among 15 cases (20\%) after OWHTO performed using FlexitSystem. All three cases of pin back out were detected in hinge fractures; otherwise, no significant differences were detected on X-ray imaging parameters, such as correction angle, pre- and postoperative $\% \mathrm{MA}, \mathrm{MPTA}$, and PTS, regardless of the occurrence of back out (Table 1). Moreover, these 15 surgeries were not performed by 

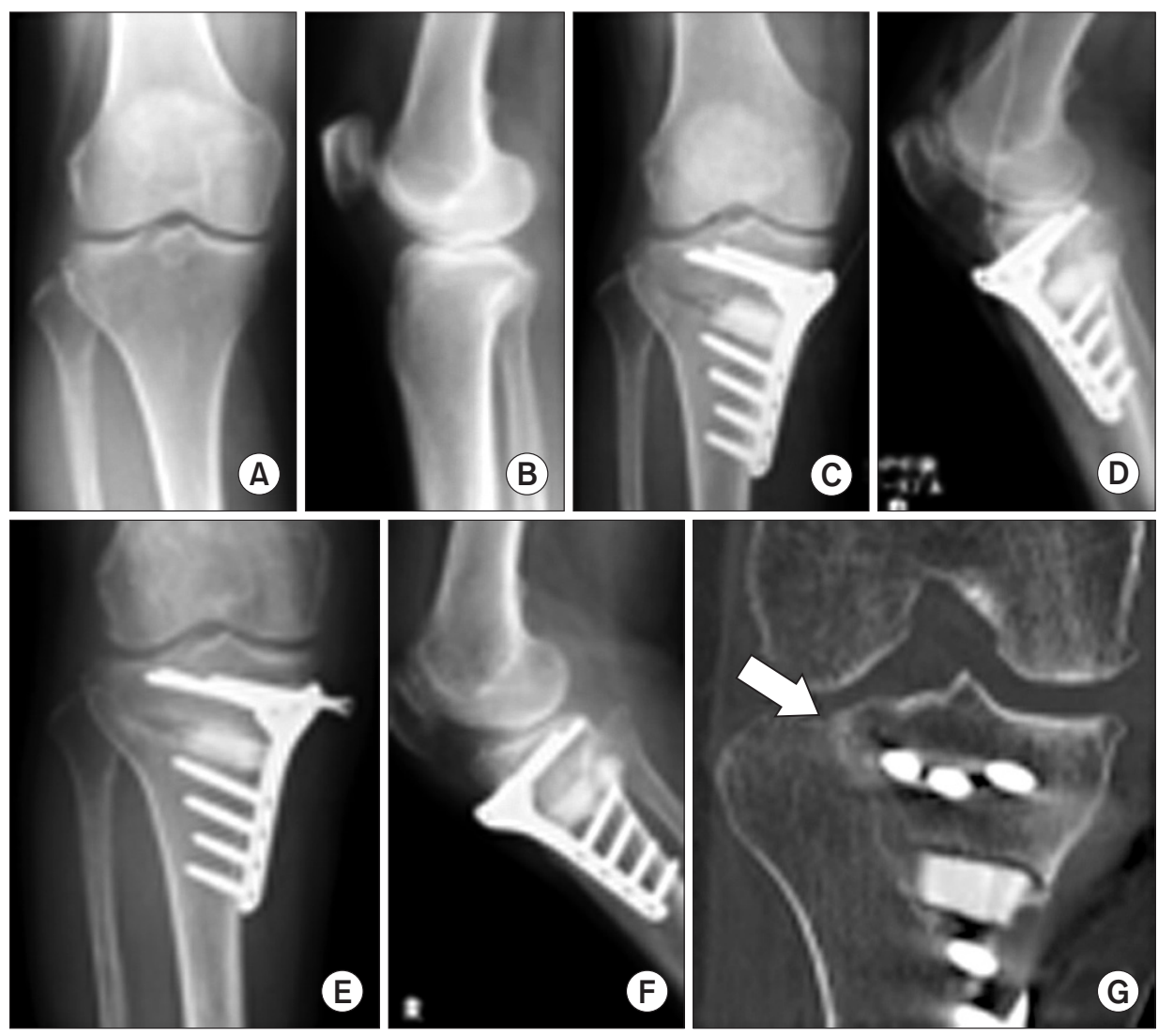

Fig. 2. (A, B) Preoperative X-rays of a 65-year-old female (case 2). (C, D) Postoperative $\mathrm{X}$-rays. (E, F) X-rays at postoperative week 2 showing locking pin back out. (G) Type III hinge fracture in computed tomography (arrow).
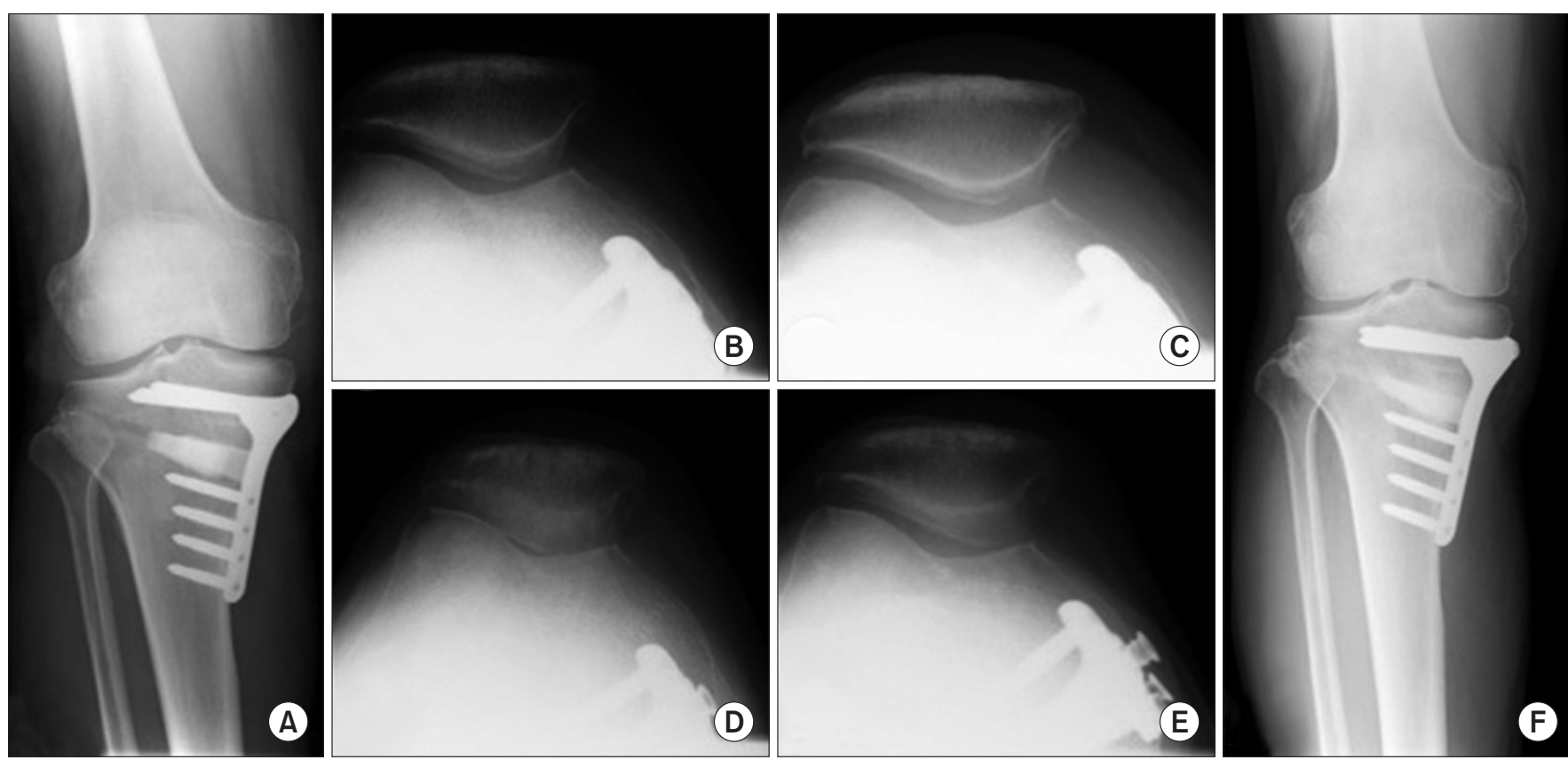

Fig. 3. (A, B) A 68-year-old male who underwent opening wedge high tibial osteotomy with a type I fracture. (C) One month after surgery, the locking pin seemed not to have backed out and he could walk with full weight bearing without pain. (D) Two months after surgery, the center pin at the proximal plate backed out. (E, F) The proximal posterior screw was also detected to have backed out without correction loss at 15 months after surgery. 

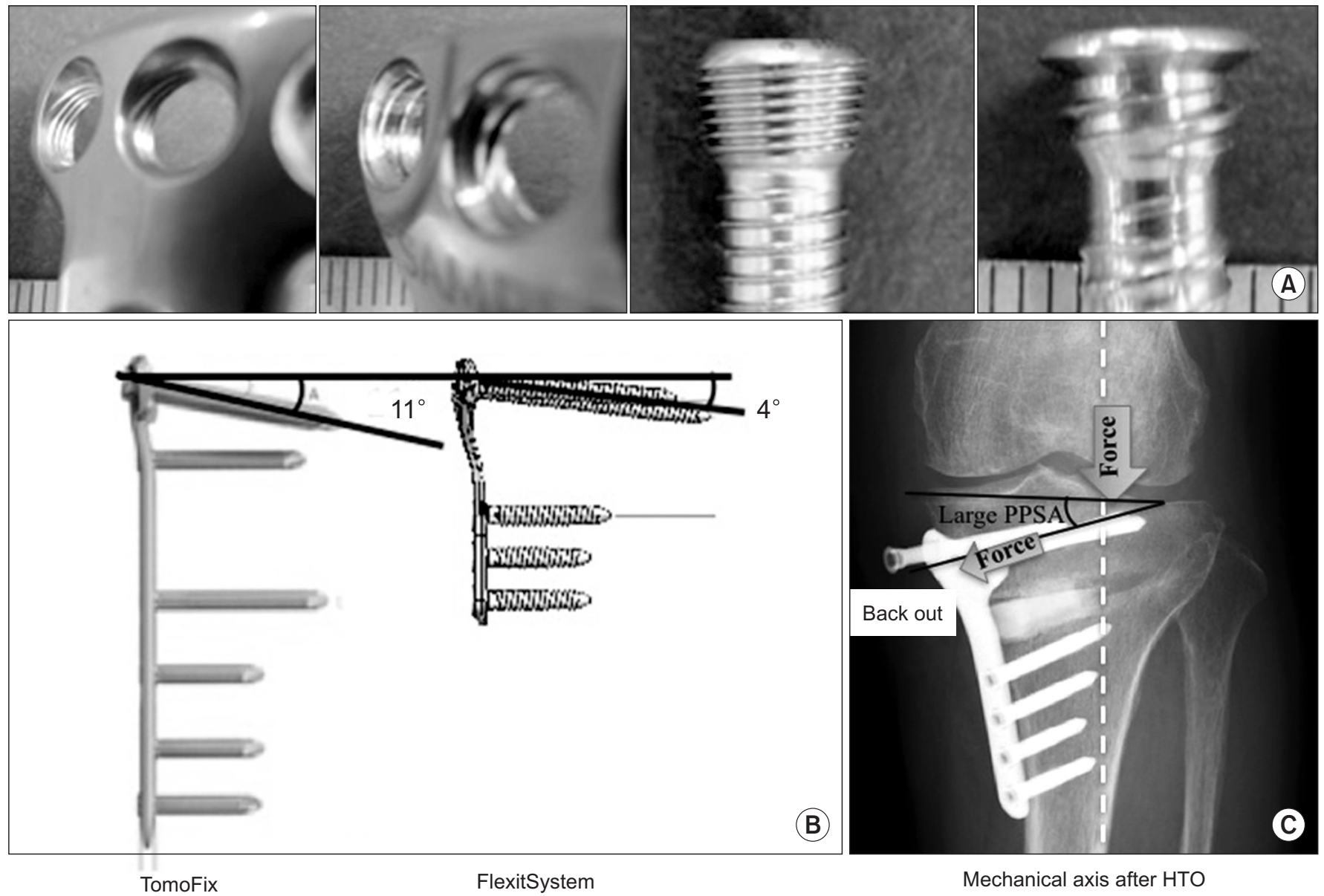

Fig. 4. (A) Comparison of locking plate systems: TomoFix (DePuy Synthes) and FlexitSystem (Neosteo). The number of threads at the plate and the screw head for the locking system is over 5 in TomoFix, which is larger than that in FlexitSystem. (B) The angle between the plate and the proximal screw was $11^{\circ}$ in TomoFix, which was larger than $4^{\circ}$ in FlexitSystem. (C) Mechanism of screw deviation with FlexitSystem. The mechanical axis after opening wedge high tibial osteotomy (HTO) passes the lateral eminence of the tibia, which is close to the tip of the locking pin. The large tibial plateau and proximal screw angle (PPSA) with FlexitSystem causes more stress than the small PPSA at the tip of the pin area. Moreover, hinge fracture may induce instability and pin back out.

the same surgeon, indicating that this specific complication was not due to a specific surgeon. Lateral cortex fractures are known to induce instability during osteotomy ${ }^{9}$, and a proximal locking system places additional mechanical stress in case of hinge fracture. No hinge fractures may prevent locking pin back out.

On the other hand, the plate system may be associated with the complication. First, the plate thickness of this low-profile locking system is $2.8 \mathrm{~mm}$. This implant is minimally invasive under the skin; however, this thin plate may cause some issues in the locking system because the number of threads at the screw head is two in FlexitSystem, compared to 5 threads in TomoFix (Fig. 4A). Second, the angle between the plate and the proximal screw might be a critical factor for screw back out. The tibial plateau and proximal screw angle (PPSA) in cases with locking pin back out was larger than that in cases without back out $(\mathrm{p}<0.001)$ (Table 1); this negatively correlated with the screw-plate angle, which was $11^{\circ}$ in TomoFix, larger than $4^{\circ}$ in FlexitSystem (Fig. 4B). Large PPSA might be associated with locking pin back out. When the correction angle was increased, PPSA became larger and proximal pins could not reach the lateral tibia. The weight bearing line passes close to the tip of the proximal pin and a hinge fracture may receive additional stress at the tip of the proximal pin, which accelerates locking pin back out (Fig. 4C). In addition, FlexitSystem was made using TA6V titanium, which is a more solid and strong material than TomoFix using pure titanium. A very strong locking plate system might induce additional force around the locking plate system because the solid plate may not be bended under mechanical stress and not work as a shock absorber. In conclusion, care should be taken when selecting a locking plate system, and a low-profile system can cause additional complications. 
Table 1. Correction with Opening Wedge High Tibial Osteotomy, Type of Hinge Fracture, and Plateau Proximal Screw Angle Compared with and without Screw Pin Back out

\begin{tabular}{lccc}
\hline \multicolumn{1}{c}{ Locking pin back out } & $(-)$ & $(+)$ & $\mathrm{p}$-value \\
\hline Correction angle $\left(^{\circ}\right)$ & $8.2 \pm 2.5$ & $8.3 \pm 1.5$ & $\mathrm{~N} / \mathrm{S}$ \\
\%MA & & & \\
Preop & $27.6 \pm 4.1$ & $20.1 \pm 8.6$ & $\mathrm{~N} / \mathrm{S}$ \\
Postop & $62.4 \pm 6.1$ & $61.7 \pm 1.5$ & $\mathrm{~N} / \mathrm{S}$ \\
MPTA $\left(^{\circ}\right)$ & & & \\
Preop & $83.8 \pm 1.7$ & $83.7 \pm 3.1$ & $\mathrm{~N} / \mathrm{S}$ \\
Postop & $92.6 \pm 1.6$ & $92.0 \pm 2.6$ & $\mathrm{~N} / \mathrm{S}$ \\
PTS $\left(^{\circ}\right)$ & & & \\
Preop & $9.6 \pm 3.0$ & $7.0 \pm 3.0$ & \\
Postop & $9.2 \pm 4.0$ & $7.7 \pm 2.5$ & \\
Type of hinge fracture & no: $7, \mathrm{I}: 5$ & $\mathrm{I}: 1, \mathrm{III}: 2$ & \\
PPSA $\left(^{\circ}\right)$ & $10.2 \pm 1.5$ & $16.2 \pm 2.1$ & $<0.001$ \\
\hline
\end{tabular}

N/S: no significance, \%MA: percentage of mechanical axis, Preop: preoperative, Postop: postoperative, MPTA: medial proximal tibial angle, PTS: posterior tibial slope, PPSA: plateau and proximal screw angle.

\section{Conflict of Interest}

No potential conflict of interest relevant to this article was reported.

\section{References}

1. Lee DC, Byun SJ. High tibial osteotomy. Knee Surg Relat Res. 2012;24:61-9.

2. Lobenhoffer P, Agneskirchner JD. Improvements in surgical technique of valgus high tibial osteotomy. Knee Surg Sports Traumatol Arthrosc. 2003;11:132-8.

3. Stoffel K, Stachowiak G, Kuster M. Open wedge high tibial osteotomy: biomechanical investigation of the modified Arthrex Osteotomy Plate (Puddu Plate) and the TomoFix Plate. Clin Biomech (Bristol, Avon). 2004;19:944-50.

4. Staubli AE, De Simoni C, Babst R, Lobenhoffer P. TomoFix: a new LCP-concept for open wedge osteotomy of the medial proximal tibia: early results in 92 cases. Injury. 2003;34 Suppl 2:B55-62.

5. Miller BS, Downie B, McDonough EB, Wojtys EM. Complications after medial opening wedge high tibial osteotomy. Arthroscopy. 2009;25:639-46.

6. Kim KJ, Song EK, Seon JK, Seol JH. Biomechanical study of the fixation plates for opening wedge high tibial osteotomy. Knee Surg Relat Res. 2015;27:181-6.

7. Schroter S, Gonser CE, Konstantinidis L, Helwig P, Albrecht D. High complication rate after biplanar open wedge high tibial osteotomy stabilized with a new spacer plate (Position HTO plate) without bone substitute. Arthroscopy. 2011;27: 644-52.

8. Martin R, Birmingham TB, Willits K, Litchfield R, Lebel ME, Giffin JR. Adverse event rates and classifications in medial opening wedge high tibial osteotomy. Am J Sports Med. 2014;142:1118-26.

9. Takeuchi R, Ishikawa H, Kumagai K, Yamaguchi Y, Chiba N, Akamatsu Y, Saito T. Fractures around the lateral cortical hinge after a medial opening-wedge high tibial osteotomy: a new classification of lateral hinge fracture. Arthroscopy. 2012;28:85-94. 